\title{
EFECTO ECOTOXICOLÓGICO DEL METOMILO EN Corydoras LACÉPÈDE, 1803 (SILURIFORMES: CALLICHTHYIDAE) Y SU CARACTERIZACIÓN LEUCOCITARIA
}

\section{ECOTOXICOLOGICAL EFFECT OF METHOMYL ON Corydoras LACÉPÈDE, 1803 (SILURIFORMES: CALLICHTHYIDAE) AND ITS LEUKOCYTE CHARACTERIZATION}

\author{
José Iannacone $e^{1,2}$ y Lorena Alvariño ${ }^{2}$
}

\section{Resumen}

El metomilo es un carbamato inhibidor de la acetilcolinesterasa que actúa por contactosistémico y es uno de los agroquímicos de mayor uso como insecticida-acaricida para el control de un amplio rango de plagas agrícolas. En el Perú es escasa la información publicada de aspectos hematológicos de osteictios para su uso potencial como biomarcador fisiológico de respuesta subletal en bioensayos ecotoxicológicos. Los objetivos del presente trabajo fueron: 1) evaluar el efecto ecotoxicológico agudo del metomilo sobre el pez amazónico ornamental Corydoras arcuatus Elwin, 1939 (Callichthyidae) a 2, 4, 6, 8, 24 y 48 h de exposición, y 2) caracterizar los leucocitos de C. arcuatus, Corydoras leucomelas Eigenmann \& Allen, 1972 y Corydoras leopardus Myers, 1933, y determinar los valores de referencia cuantitativos. Los bioensayos con metomilo se realizaron en envases plásticos de $1000 \mathrm{~mL}$. Se encontraron valores de $\mathrm{CL}_{50}(\mathrm{mg}$ IA $\mathrm{L}^{-1}$ ) de $10.84 ; 3.18 ; 0.28 ; 0.01 ; 0.002$ y $<0.002$ a 2, 4, 6, 8, 24 y 48 h, respectivamente. La $\mathrm{TL}_{50}$ a la concentración más baja de $3.2 \mathrm{mg} \mathrm{L}^{-1}$ fue $4.83 \mathrm{~h}$. La extracción de la sangre, se llevó a cabo por el método de punción cardiaca, realizándose luego extensiones teñidas con Giemsa y con Wright. No se encontraron diferencias en los recuentos leucocitarios observados en ambos procedimientos de tinción, pero con ligeras diferencias entre sexos. No se encontraron diferencias significativas entre las especies de Corydoras examinadas. Cuantitativamente, se encontró la siguiente secuencia: linfocitos $(64.6 \%)>$ monocitos $(18.4 \%)>$ neutrófilos $(8.2 \%)>$ eosinófilos $(5.2 \%)>$ basófilos (3.2\%). Los parámetros hematológicos obtenidos son un aporte al conocimiento de este género y pudieran potencialmente ser empleados como biomarcadores de respuesta subletal para los bioensayos ecotoxicológicos.

Palabras claves: Metomilo, efecto letal, riesgo ambiental, Amazonía, Corydoras, leucocitos, parámetros hematológicos

\begin{abstract}
Methomyl is a carbamate acetylcholinesterase inhibitor that acts by contact and systemically and is one of the agrochemicals of mayor use as insecticide-acaricide to control a broad spectrum of agricultural pests. Peru lacks information regarding hematological aspects of bony fishes which could be used as potential physiological biomarkers of sub lethal responses in ecotoxicological bioassays. Aims of the current research were: 1) evaluate acute toxicological effect of methomyl on an ornamental Amazonian fish Corydoras arcuatus Elwin, 1939 (Callichthyidae) at 2, 4, 6, 8, 24 and $48 \mathrm{~h}$ exposure, and 2) characterize leukocytes of C. arcuatus, Corydoras leucomelas Eigenmann \& Allen, 1972 and Corydoras leopardus Myers, 1933, and determinate quantitative reference values. Bioassays with methomyl were performed on plastic pots of $1000 \mathrm{~mL}$. Values of $\mathrm{LC}_{50}\left(\mathrm{mg} \mathrm{AI} \mathrm{L}{ }^{-1}\right)$ were $10.84 ; 3.18 ; 0.28 ; 0.01 ; 0.002$ and $<0.002$ at 2, 4, 6, 8, 24 and $48 \mathrm{~h}$, respectively. $\mathrm{LT}_{50}$ at the lower concentration of $3.2 \mathrm{mg} \mathrm{L}^{-1}$ was $4.83 \mathrm{~h}$. Blood extraction, was done by the cardiac puncture method, and smears were stained with Giemsa and Wright. Differences on leukocytes counts were not observed in both staining procedures, but slight differences between sexes were found Non significant differences between species of Corydoras were observed. Quantitatively, the following sequence was found: lymphocytes $(64.6 \%)>$ monocytes $(18.4 \%)>$ neutrophiles $(8.2 \%)>$ eosinophiles $(5.2 \%)>$ basophiles $(3.2 \%)$. Blood parameters obtained contribute to the knowledge of this genus and could be potentially employed as biomarkers of sublethal responses in ecotoxicological bioassays.
\end{abstract}

Key Words: Amazonía, Corydoras, lethal effect, methomyl, leukocytes, hematological parameters, environmental risk 


\section{Introducción}

El metomilo es un carbamato inhibidor de acetilcolinesterasa que actúa por contacto-sistémico (Strickman, 1985; Farré et al., 2002; Michaud \& Grant, 2003; Li et al., 2008) y es uno de los agroquímicos de mayor uso como insecticidaacaricida en el Perú para el control de un amplio rango de plagas agrícolas (Morishita 2001; El-Aw et al., 2008). Es usado también como molusquicida, garrapaticida y contra arañas; así como en cebos para el control de la mosca doméstica. La mayoría de sus productos comerciales están clasificados por la USEPA (Agencia de Protección Ambiental de la Estados Unidos) como clase I. El metomilo en el Perú es clasificado como extremadamente tóxico Clase Ib. Existen evidencias que el metomilo es un plaguicida que tiene efecto como Disruptor endocrino (DE). La dosis de aplicación de metomilo para el control de plagas en la agricultura es como promedio de $1.35 \mathrm{~g}$ IA L ${ }^{-1}$ (Panger \& Echevarría, 2007).

La ictiohematología es una disciplina que estudia la sangre en los peces, que incluye a la célula sanguínea desde la perspectiva morfológica, bioquímica $\mathrm{y}$ funcional $\mathrm{y}$ a los órganos hematopoyéticos, las enfermedades relacionadas y cualquier fenómeno o patología que relacione las células y sus órganos productores (Valenzuela et al., 2003, 2008). Las oscilaciones en las condiciones abióticas como los tóxicos causan modificaciones fisiológicas en los niveles de algunos parámetros sanguíneos (Centeno et al., 2007; Valenzuela et al., 2002). En adición, se ha determinado que estos parámetros pueden estar también influenciados por factores tales como la especie, la edad, el fotoperíodo, el estado nutricional y la metodología empleada (Hrubec et al., 2000; Iannacone et al., 2006a,b; Centeno et al., 2007). La variación de los parámetros hematológicos pueden ser utilizados como indicadores de contaminación (Landman et al., 2005).

Corydoras Lacépède, 1803 (Siluriformes: Callichthyidae) conocido como "basurerito", "limpia fondos" o "shirui", es un pez amazónico característico de aguas limpias (Britto \& Lima, 2003). Es un género bentónico de corrientes lentas y de fondos arenosos. Las especies más importantes del género tienen importancia en acuarofilia o como peces ornamentales (Kaatz \& Lobel, 1999; Ortiz \& Iannacone, 2008). Corydoras tiene 143 especies validas (Reis, 2003; Artoni et al., 2006), de las cuales 26 se han registrado para el Perú. Está distribuido en Sudamerica en una diversidad de hábitats. En Corydoras paleatus Jenyns, 1842, se ha evaluado la toxicidad del fenitrotion, metilparation y de las microcistinas (Fanta et al., 2003; Sarikaya et al., 2004; Cazenave et al., 2006).

Los objetivos del presente trabajo fueron: 1) evaluar el efecto toxicológico letal del metomilo sobre el pez amazónico ornamental Corydoras arcuatus Edwin, 1939 (Callichthyidae) a 2, 4, 6, 8, 24, $48 \mathrm{~h}$ de exposición, y 2) determinar los valores de referencia cuantitativos de los leucocitos de C. arcuatus, Corydoras leucomelas Eigenmann \& Allen, 1972 y Corydoras leopardus Myers, 1933.

\section{Materiales y métodos}

$\underline{\text { Metomilo }}$

CAS $=16752-77-5$. La formulación de polvo soluble (PS) del metomilo, S-metil N(metilcarbamoyloxi) tioacetimidato $\left(\mathrm{C}_{5} \mathrm{H}_{10} \mathrm{~N}_{2} \mathrm{O}_{2} \mathrm{~S}\right.$, PM $=162,21)$ empleada, fue representativa del mercado Nacional Peruano (Lannate ${ }^{\circledR}$ 90, Categoría Ib, extremadamente tóxica). El metomilo presenta una toxicidad aguda oral en ratas de $\mathrm{DL}_{50}=17 \mathrm{mg} \mathrm{Kg}^{-1} \mathrm{y}$ una toxicidad aguda dermal de $\mathrm{DL}_{50}>5000 \mathrm{mg} \mathrm{Kg}^{-1}$. Su punto de ebullición es $79^{\circ} \mathrm{C}$, su presión de vapor es $6.65 \mathrm{mPa}$ a $25^{\circ} \mathrm{C}$, su constante de la Ley de Henry fue $1.8 \times 10-10 \mathrm{~atm} \mathrm{~m}^{3} / \mathrm{mol}$, su coeficiente de partición octanol-agua $(\mathrm{Kow})=1.29-1,33$, su densidad a $20^{\circ} \mathrm{C}$ es de 1.3 , su solubilidad en agua es de $57.9 \mathrm{~g} \mathrm{~L}^{-1}$ a $25^{\circ}$ $\mathrm{C}$, no es reactiva, no es corrosiva y no es volátil. $\mathrm{Su}$ GUS (Grado de difusión a las aguas subterráneas) indica un valor intermedio de 1.57 . No es considerado mutagénico, teratogénico o carcinogénico. Su dosis de aplicación promedio en los ecosistemas agrícolas es de $0.22-1.8 \mathrm{lb} \cdot$ acre $^{-1}$.

Bioensayos ecotoxicologicos

Los especimenes adultos de C. arcuatus "shirui" procedentes de Iquitos, Loreto, Perú fueron obtenidos de un acuario del distrito de Breña, Lima, Perú. Cuatrocientos peces fueron trasladados al laboratorio de Ecofisiología Animal de la Facultad de Ciencias Naturales y Matemática de la Universidad Nacional Federico Villarreal en baldes de plástico de $4 \mathrm{~L}$ de capacidad. Antes de la aclimatación en el laboratorio se descartaron los individuos que hubieran sufrido daños mecánicos durante el transporte. Los peces adultos fueron colocados una semana previa a las pruebas ecotoxicológicas en acuarios de vidrio de 90 $\mathrm{cm}$ de largo x $40 \mathrm{~cm}$ ancho x $30 \mathrm{~cm}$ de alto, en agua de grifo declorinada de Lima, Perú reposada y oxigenada (Oxígeno disuelto $>8 \mathrm{mg} \mathrm{L}^{-1}$ ) (Castillo, 2004). Se implantó un calentador en el acuario para alcanzar la temperatura de $25-26^{\circ} \mathrm{C}$. Los parámetros físicos como la temperatura y el $\mathrm{pH}$ (7.6-7.9) del cultivo parcial se midieron tres veces por semana. El fotoperiodo fue aproximadamente de 12:12. Los peces seleccionados presentaron una apariencia externa saludable para su uso en los ensayos toxicológicos y presentaron los siguientes parámetros biométricos: Longitud total $(\mathrm{cm})=4.81 \pm 0.29$; longitud estándar $(\mathrm{cm})=3.88 \pm 0.25$, y peso $(\mathrm{g})=3.08 \pm 0.36$. Los peces no fueron alimentados al menos $24 \mathrm{~h}$ antes del experimento, ni tampoco durante el experimento con el objetivo de mantener una adecuada calidad del agua. Las pruebas de toxicidad se realizaron con cuatro repeticiones, y cinco concentraciones más el control. Se emplearon cinco concentraciones de 
metomilo (IA): $3,2 \mathrm{mg} \mathrm{IA} \mathrm{L}^{-1}, 16 \mathrm{mg} \mathrm{IA} \mathrm{L}^{-1}, 80 \mathrm{mg}$ IA $\mathrm{L}^{-1}, 400 \mathrm{mg} \mathrm{IA} \mathrm{L}^{-1}$ y $2000 \mathrm{mg} \mathrm{IA} \mathrm{L}^{-1}$. Se empleó un factor de dilución de 0.2. Los bioensayos se realizaron en envases plásticos de $1000 \mathrm{~mL}$ a los que se transfirieron 5 individuos y se realizaron lecturas a 2 , $4,6,8,24,48$ h de exposición. Se usó como criterio de mortalidad la carencia de movilidad. Los peces muertos fueron removidos inmediatamente. La eficacia de los tratamientos y las repeticiones se evaluó a través de un análisis de varianza (ANDEVA) de dos vías, previa transformación de los datos a raíz cuadrada del arcoseno. En el caso de existir diferencias significativas entre los tratamientos y entre las repeticiones se realizó la prueba de Tukey.

Los cálculos de la mortalidad corregida se realizaron mediante la fórmula de Abbott en caso de muerte natural en el testigo cuando la mortalidad fue menor al 20\% (Iannacone \& Alvariño, 2007) Los valores de $\mathrm{CL}_{50}$ (Concentración letal media) en $\mathrm{mg} \mathrm{L}^{-1}$ y $\mathrm{TL}_{50}$ (Tiempo letal medio) en $\mathrm{h}$ fueron calculados usando el programa computarizado Probit versión 1.5 (Weber, 1993). El modelo de regresión fue verificado usando el Chi-cuadrado. Se emplearon los modelos GENEEC (Parker et al., 1995), PRZM-2 (Carousel et al., 2005) y EXAMII (Burns, 2004) para determinar la CAE (concentración ambiental esperada) del metomilo en el agua, y se calculó el cociente de riesgo (CR) en el ambiente acuático (Iannacone et al., 2007; Panger \& Echevarría, 2007).

Caracterización leucocitaria

34 individuos de Corydoras exportados como ornamentales fueron obtenidos de un acuario de Jesús María, Lima, Perú: C. leucomelas $(\mathrm{n}=26), C$. arcuatus $(\mathrm{n}=1)$ y C. leopardus $(\mathrm{n}=7)$. Los peces fueron aclimatados en acuarios de $60 \mathrm{~cm} \times 20 \mathrm{~cm} \times 30$ $\mathrm{cm}$ por $48 \mathrm{~h}$ en el laboratorio, en agua de grifo declorinada de Lima, Perú con las características físico-químicas señaladas por Iannacone et al. (2006b). A cada ejemplar se le determinó el sexo (machos, hembras o indiferenciados), se registró su longitud total $(\mathrm{cm})$, longitud estándar $(\mathrm{cm})$, peso corporal ( $\mathrm{P}$ en $\mathrm{g}$ ). Posteriormente se les anestesió con Lidocaína al $2 \%$ (Xilocaína ${ }^{\circledR}$ ), y se realizó la extracción de la sangre mediante el método de punción cardiaca, realizándose luego extensiones teñidas con Giemsa y con Wright, y el recuento leucocitario a un microscopio óptico con aumento de 1000x en un total de 100 células (Orün \& Erdemil, 2002). Para todos los parámetros analizados se determinó su media y su desviación estándar. Los análisis estadísticos se realizaron para los 34 individuos de Corydoras, para C. leucomelas y para C. leopardus. No se efectuaron para C. arcuatus por solo evaluarse un solo individuo. Se empleó el coeficiente de correlación de Pearson (r) entre los parámetros biométricos de Corydoras y el conteo leucocitario. Se comparó si el número de los diferentes leucocitos eran diferentes entre los machos, hembras e indiferenciados empleando la prueba de ANDEVA y la prueba de Tukey. En adición, se usó la prueba de t de student para datos pareados para comparar si existen diferencias entre el recuento leucocitario entre la coloración Giemsa y Wright (Iannacone et al., 2006b). La prueba t de student y la prueba de Levene fueron empleadas para determinar si existían diferencias entre los parámetros biométricos y el conteo leucocitario de C. leucomelas y C. leopardus. No se determinaron las diferencias significativas en la caracterización leucocitaria entre hembras, machos e indeferenciados para cada especie de Corydoras por separado debido al bajo número de especimenes en alguna de las tres categorías.

Se usó el paquete estadístico SPPS, versión 15.00 para el cálculo de los todos los estadísticos descriptivos e inferenciales.

\section{Resultados y Discusión}

Bioensayos ecotoxicologicos con metomilo

Los porcentajes de mortalidad de C. arcuatus a las cinco concentraciones de metomilo y a los seis periodos de exposición es indicado en la Tabla 1. Se encontraron los siguientes valores de $\mathrm{CL}_{50}(\mathrm{mg}$ de metomilo $\mathrm{L}^{-1}$ ) de $10.84 ; 3.18 ; 0.28 ; 0.01 ; 0.002 \mathrm{y}<$ 0.002 a 2, 4, 6, 8, 24 y $48 \mathrm{~h}$, respectivamente sobre $C$. arcuatus. $\mathrm{La} \mathrm{TL}_{50}$ a la concentración más baja de 3,2 $\mathrm{mg} \mathrm{L}^{-1}$ fue $4,83 \mathrm{~h}$. La Tabla 2 que incluye a $C$. arcuatus, muestra que esta especie resultó ser altamente sensible al metomilo en comparación a otras 28 especies dulceacuícolas (10 peces; 9 crustáceos; 4 insectos y 5 moluscos) (Munn et al., 2006; Li et al., 2008). Se observó que los insectos presentaron mayor sensibilidad que los crustáceos, y éstos a su vez que los peces, y éstos últimos que los moluscos $(\mathrm{F}=4.90$; $\mathrm{P}=0.01$ ) (Tabla 2).

De acuerdo a la clasificación de la toxicidad de los pesticidas en los organismos acuáticos, el metomilo es clasificado como un plaguicida altamente tóxico ( $\mathrm{Li}$ et al., 2008). La toxicidad del metomilo en peces varía entre $320 \mathrm{mg} \cdot \mathrm{L}^{-1}$ para Ictalurus punctatus y 5425 $\mathrm{mg} \cdot \mathrm{L}^{-1}$ para Oncorhynchus clarki (Tabla 2). C. arcuatus es 160 y 2713 veces más sensible al metomilo que I. punctutus y O. clarki.

La mayor toxicidad y sensibilidad al metomilo encontrada en C. arcuatus en términos de $\mathrm{CL}_{50}$ en comparación a las otras 28 especies dulceacuícolas podría deberse a que esta especie de pez amazónico del Neotrópico carece de un adecuado sistema de detoxificación del metomilo, el cual inhibe la actividad de la acetilcolina (AchE) y de la glutation Stransferasa (GTSs) produciendo una alta sensibilidad a este carbamato, como ha sido observado en otras especies de peces (Li et al., 2008).

Al realizar una evaluación de riesgo del metomilo empleado a C. arcuatus, se muestran según el modelo GENEEC para determinar la CAE (concentración ambiental esperada) valores de 409.386 y $350 \mathrm{ug} \cdot \mathrm{L}^{-1}$ a 
un nivel pico, 21 días y 56 días, respectivamente (Panger \& Echevarría, 2007). Por ende, el cociente de riesgo (CR) fue de 204.5; 193 y 175 a un nivel pico, 21 días y 56 días, respectivamente. De igual manera al usar el procedimiento PRZM-2/EXAMII para determinar la CAE, se obtuvieron valores de 99, 95, 85 y $79 \mathrm{ug} \cdot \mathrm{L}^{-1}$ a un nivel pico, 21 días, 56 días y 90 días, respectivamente (Panger \& Echevarría, 2007). Así, al aplicar el CR, se obtuvieron valores de 49.5; 47.5; 42.5 y 39.5 a un nivel pico, 21 días, 56 días y 90 días, respectivamente. De esta manera, ambos modelos GENEEC y PRZMZ/EXAMII que simulan el destino y transporte del metomilo en el ambiente acuático evidencian según los $\mathrm{CR}$ que presentaría un alto riesgo acuático para las comunidades ícticas neotropicales. Sin embargo, los valores de Kow del metomilo nos señalan una baja tendencia a la bioacumulación en peces.

Caracterizacion leucocitaria

La Tabla 3 indica tres parámetros biométricos (longitud total, longitud estándar y peso), de sexo (macho, hembra e indiferenciado) y de recuento leucocitario (linfocitos, monocitos, neutrófilos, eosinófilos y basófilos) por Giemsa y Wright para Corydoras spp., C. leucomelas, C. arcuatus y $C$. leopardus.

Se observaron diferencias en la longitud total, longitud estándar y el peso de los peces machos, hembras e indiferenciados de Corydoras spp. (Tabla 4). Observándose que las hembras presentaron mayor longitud total, longitud estándar y peso que los machos e indiferenciados (Tabla 4). No se encontraron diferencias significativas en el recuento leucocitario de linfocitos, monocitos, eosinófilos y basófilos entre los peces machos, hembras e indiferenciados de Corydoras spp. Solo se vio diferencias entre el número de neutrófilos por Giemsa y Wright con relación al sexo de los peces (Tabla 4).

Al comparar la longitud total, el peso y el recuento leucocitario (linfocitos, monocitos, neutrófilos, eosinófilos y basófilos) no se encontraron diferencias significativas entre $C$. leucomelas y $C$. leopardus $(\mathrm{t}=$ 0.09-1.83; $\mathrm{P}=0.07-0.92$; Prueba de Levene $\mathrm{W}=0.11$ 4.20; $\mathrm{P}=0.06-0.74)$. Solo se observó diferencias significativas entre la longitud estándar de $C$. leucomelas $(3.56 \pm 0.4 \mathrm{~cm})$ y C. leopardus $(4.17 \pm$ $0.52 \mathrm{~cm})(\mathrm{t}=3.30 ; \mathrm{P}=0.002)$.

Según la prueba de $t$ de Student no se encontraron diferencias significativas entre el número de linfocitos, monocitos, neutrófilos, eosinófilos y basófilos evaluados mediante la técnica de coloración de Giemsa y Wright (Tabla 5). La longitud total solo se vio correlacionada positivamente con el número de linfocitos $(\mathrm{r}=0.46 ; \mathrm{P}=0.009)$ y negativamente con el número de neutrófilos $(\mathrm{r}=-36 ; \mathrm{P}=0.04)$.

Los leucocitos, en general, presentaron características similares a las descritas para otros peces amazónicos. Las células sanguíneas de los frotis que se analizaron en las lecturas fueron similares morfológicamente a las descritas para Shroedenchthys chilensis (Guichenot 1848) (Filho et al., 1992; Valenzuela et al., 2003), Salminus maxillosus Valenciennes 1840 (Veiga et al., 2000; Ranzani-Paiva et al., 2003), Chaetodipterus faber (Broussonet 1782) (Bastardo \& Barberán, 2004) y Astronotus ocellatus (Agassiz 1831) (Iannacone et al., 2006a). En el recuento se observó un predominio de los linfocitos, lo cual es concordante con lo encontrado en otros peces teleósteos como Pimelodus maculatus Lacèpéde 1803, Synbranchus marmoratus (Bloch 1795), Mugil platanus Günther 1880, Oncorhynchus mykiss (Walbaum 1792), A. ocellatus, Pterophyllum scalare (Lichtenstein 1823) y Salminus affinis (Steindachner 1880) (Örün \& Erdemil, 2002; Ranzani-Paiva et al., 2003; Valenzuela et al., 2003; Iannacone et al., 2006a,b; Atencio-García et al., 2007). Se encontraron ligeras diferencias en los recuentos leucocitarios observados en ambos procedimientos de tinción y entre sexos. No se encontraron diferencias significativas entre las tres especies de Corydoras examinadas. Cuantitativamente, se encontró la siguiente secuencia: linfocitos $(64.6 \%)>$ monocitos $(18.4 \%)>$ neutrófilos $(8.2 \%)>$ eosinófilos $(5.2 \%)>$ basófilos $(3.2 \%)$. Los parámetros hematológicos obtenidos en Corydoras spp. son un aporte al conocimiento de este género íctico y pudieran potencialmente ser empleados como biomarcadores de respuesta subletal en bioensayos ecotoxicológicos.

\section{Literatura citada}

Artoni R.F., Terêncio M.L., Vicari M.R., Matiello M.C.A., Cestari M.M. \& Bertollo L.A. C. 2006. Cytogenetics of two sympatric Corydoras species (Pisces, Siluriformes, Callichthyidae) of southern Brazil. Brazilian Journal of Biology. 66: 191-198.

Atencio-García V., Genes L.F., Madariaga M.D. \& Pardo C.S. 2007. Hematología y química sanguínea de juveniles de rubio (Salminus affinis Pises: Characidae) del río Sinú. Acta Biológica Colombiana. 12: 27-40.

Bastardo A. \& Barberán R. 2004. Parâmetros hematológicos de la paragua, Chaetodipterus faber (Broussonet) (Pisces. Ephippidae), em condiciones de cultivo. Zootecnia Tropical. 22: 361-370.

Britto M.R. \& Lima F.C.T. 2003. Corydoras tukano, a new specie of corydoradine catfish from the rio Tiquié, upper rio Negro basin, Brazil (Ostariophysi: Siluriformes: Callichthyidae). Neotropical Ichthyology. 1: 83-91.

Burns L.A. 2004. Exposure Analysis Modeling System (EXAMS): User Manual and System Documentation. EPA600R00081.

Carousel R.F., Imhoff J.C., Hummel P.R., Cheplick J.M. \& Donigian A.S. 2005. PRZM-3, A Model for Predicting Pesticide and Nitrogen Fate in the Crop Root and Unsaturated Soil Zones: Users Manual for release 3.0. National Exposure Research Laboratory Office of Research and development U.S. Environmental Protection. U.S.A.

Castillo G. 2004. Ensayos ecotoxicológicos y métodos de evaluación de calidad de aguas. Estandarización, 
intercalibración, resultados y aplicaciones. IMTA. México.

Cazenave J., Bistoni M. de L., Pesce S.F. \& Wunderlin D.A. 2006. Differential detoxification and antioxidant response in diverse organs of Corydoras paleatus experimentally exposed to microcystin-RR. Journal of Parasitology. 92: 459-463.

Centeno L., Silva-Acuña R., Barrios R., Salazar L.R., Matute C. \& Pérez J.L. 2007. Características hematológicas de la cachama (Colossoma macropomum) em tres etapas de crecimiento cultivadas em el estado Delta Amacuro, Venezuela. Zootecnia Tropical. 25: 237-243.

El-Aw M.A.M., Draz K.A.A., Hashem A.G. \& El-Gendy I.R. 2008. Mortality comparison among spinosad-, actara-, malathin-, and methomyl- containing baits against peach fruit fly, Bactrocera zonata Saunders (Diptera: Tephritidae) under laboratory condictions. Journal of Applied Science Research. 4: 216-223.

Fanta E., Rios F.S., Romao S., Vianna A.C. \& Freiberger S. 2003. Histopathology of the fish Corydoras paleatus contaminated with sublethal levels of organophosphorus in water and food. Ecotoxicology and Environmental Safety. 54:119-130.

Farré M., Fernández J., Paez M., Granada L., Barba L., Gutierrez H.M., Pulgarin C. \& Barceló D. 2002. Analysis and toxicity of methomyl and ametryn after biodegradation. Analytical and Bioanalytical Chemistry. 373: 704-709.

Filho W., Eble G.J., Kassner G., Caprario F.X., Dafre A.L. \& Ohira M. 1992. Comparative hematology in marine fish. Comparative biochemical Physiology and comparative physiology. 102: 311-321.

Hrubec T.C., Cardinale J.L. \& Smith S.A. 2000. Hematology and plasma chemistry reference intervals for culture Tilapia (Oreochromis hybrid). Veterinary Clinical Pathology. 29: 7-12.

Iannacone J. \& Alvariño L. 2007. Ecotoxicidad acuática de dos colorantes y de tres antiparasitarios de importancia en acuicultura en Daphnia magna. Ecología Aplicada. 6: 101-110.

Iannacone J., Bello C., Hernández N. \& Díaz M. 2006a. Caracterización leucocitaria del pez amazónico Pterohyllum scalare (Lichtenstein, 1823) (Perciformes: Cichlidae) de Perú. Biotempo. 6: 32-37.

Iannacone J., Falcón W. \& Vargas R. 2006b. Parámetros hematológicos del acarahuazú Astronotus ocellatus (Agassiz, 1831) (Cichlidae: Perciformes). The Biologist (Lima). 4: 16-18.

Iannacone J., Onofre R., Huanqui O., Giraldo J., Mamani N., Miglio M. \& Alvariño L. 2007. Evaluación del riesgo ambiental del insecticida metamidofos en bioensayos con cuatro organismos acuáticos no destinatarios. Agricultura Técnica (Chile). 67:126-138.

Kaatz I.M. \& Lobel P.S. 1999. Acoustic behavior and reproduction of five species of Corydoras catfishes (Callichthyidae). The Biological Bulletin. 197: 241-242.

Landman M. J., Van Dern Heuvel M.R., Finley M., Bañon H.J. \& Ling N. 2005. Combined effects of pulp and paper effluent, dehydroabietic acid, and hypoxia on swimming performance, metabolism, and hematology of rainbow trout. Ecotoxicology and Environmental Safety. 65: 314-322.
Li H., Jiang H., Gao X., Wang X., Qu W., Lin R. \& Chen J. 2008. Acute toxicity of the pesticide methomyl on the topmouth gudgeon (Pseudoraspora parva): mortality and effects on four biomarkers. Fish Physiology and Biochemical. 34: (in press).

Michaud J.P. \& Grant A.K. 2003. IPM-compatibility of foliar insecticide for citrus: Indices derived from toxicity to beneficial insects from four orders. Journal of Insect Science. 3: 18, 10 pp. Disponible en línea: insectscience.org/3.18

Morishita M. 2001. Toxicity of some insecticides to larvae of Frankliniella occidentalis (Pergande) (Thysanoptera: Thripidae) evaluated by the petri dish-spraying tower method. Applied Entomology and Zoology. 36: 137141.

Munn M.D., Gilliom R.J., Moran P.W. \& Novell L.H. 2006. Pesticide toxicity index for freshwater aquatic organisms, 2nd Ed. Scientific Investigations Report 2006-5148. U.S. Department of the Interior Dirk Kempthorne, Secretary U.S. Geological Survey P. Patrick Leahy, Acting Director.

Ortiz N. \& Iannacone J. 2008. Estado actual de los peces ornamentales amazónicos del Perú que presentan mayor demanda de exportación. The Biologist (Lima). 6: 48 63.

Orüm I. \& Erdemil A.U. 2002. A study of blood parameters of Capoella trutta (Heckel, 1843). On line Journal of Biological Science. 2: 508-511.

Panger M.A. \& Echevarría M. 2007. Risk of methomyl use to the Federally listed California red-legged frog (Rana aurora draytonii). Environmental Fate and Effects Division Office of Pesticide Programs. Washington, D.C. 20460.

Parker R.D., Jones R.D. \& Nelson H.P. 1995. GENEEC: A Screening Model for Pesticide Environmental Exposure Assessment. Proceedings of the International Exposure Symposium on Water Quality Modeling.

Ranzani-Paiva M.J.T., Rodriguez E.L., Veiga M.L., Eiras A.C. \& Campos B.E.S. 2003. Differencial leukocyte counts in "dorado" Salminus maxillosus Valenciennes, 1840 , from the mogi-guacu river, Pirassununga, S.P. Brazilian Journal of biology. 63: 517-525.

Reis R.E. 2003. Family Callichthyidae (armored catfishes). Pp. 291-309. In: Reis, R. E., S. O. Kullander \& C. J. Ferraris (Eds.). Check List of the Freshwater Fishes of South and Central America. Porto Alegre, EDIPUCRS,

Sarikaya R., Selvi M. \& Erkoc F. 2004. Investigation of acute toxicity of fenitrothion on peppered corydoras (Corydoras paleatus) (Jenyns, 1842). Chemosphere. 56: 697-700.

Strickman D. 1985. Aquatic bioassay of 11 pesticides using larvae of the mosquito, Wyeomyia smithii (Diptera: Culicidae). Bulletin of Environmental Contamination and Toxicology. 35: 133-142.

Valenzuela A., Alveal K. \& Tarifeño E. 2002. Respuesta hematológica de truchas (Onchorhynchus mykiss Walbaum 1792) a estrés hipóxico agudo: serie roja. Gayana. 66: 255-261.

Valenzuela A., Oyarzún C. \& Silva V. 2003. Células sanguíneas de Schroederichthys chilensis (Guichenot 1848) (Elasmobranchii, Scyliorhinidae): la serie blanca. Gayana. 67: 130-136.

Valenzuela A., Silva V.M. \& Klempau A.E. 2008. Effects of different artificial photoperiods and temperatures on 
haematological parameters of rainbow trout (Onchorhynchus mykiss). Fish Physiology and Biochemical. 34: 159-167.

Veiga M.L., Egami M.I., Ranzani-Paiva M.J.T. \& Rodríguez E.L. 2000. Aspectos morfológicos y citoquímicos de las células sanguíneas de Salminus maxillosus
Valenciennes, 1840 (Characiformes, Characidae). Revista Chilena de Anatomía. 18: 245-250.

Weber C. 1993. Methods for measuring the acute toxicity of effluents and receiving waters to freshwater and marine organisms. EPA/600/4-90/027F.

\section{Tablas citadas en el texto}

Tabla 1. Porcentaje de mortalidad de C. arcuatus expuesta al metomilo.

\begin{tabular}{ccccccc}
\hline $\mathbf{m g}^{-\mathbf{L}^{-1}}$ & $\mathbf{2 ~ \mathbf { ~ }}$ & $\mathbf{4} \mathbf{h}$ & $\mathbf{6 ~ h}$ & $\mathbf{8 ~ h}$ & $\mathbf{2 4} \mathbf{h}$ & $\mathbf{4 8 ~ h}$ \\
\hline 0 & $0 \mathrm{a}$ & $0 \mathrm{a}$ & $0 \mathrm{a}$ & $0 \mathrm{a}$ & $0 \mathrm{a}$ & $0 \mathrm{a}$ \\
3.2 & $13.3 \mathrm{a}$ & $33.3 \mathrm{~b}$ & $66 \mathrm{~b}$ & $80 \mathrm{~b}$ & $81.5 \mathrm{~b}$ & $86.6 \mathrm{~b}$ \\
16 & $80 \mathrm{~b}$ & $86.6 \mathrm{c}$ & $86.6 \mathrm{c}$ & $86.6 \mathrm{bc}$ & $86.6 \mathrm{bc}$ & $95 \mathrm{~b}$ \\
80 & $81.5 \mathrm{~b}$ & $87.5 \mathrm{c}$ & $87.5 \mathrm{c}$ & $87.5 \mathrm{bc}$ & $90 \mathrm{bc}$ & $100 \mathrm{~b}$ \\
400 & $85 \mathrm{~b}$ & $90 \mathrm{c}$ & $90 \mathrm{c}$ & $90 \mathrm{bc}$ & $90 \mathrm{bc}$ & $100 \mathrm{~b}$ \\
2000 & $87.5 \mathrm{~b}$ & $93.7 \mathrm{c}$ & $100 \mathrm{c}$ & $100 \mathrm{c}$ & $100 \mathrm{c}$ & $100 \mathrm{~b}$ \\
\hline
\end{tabular}

Letras minúsculas iguales en una misma columna indican que los promedios son estadísticamente iguales ( $\mathrm{p} \geq 0.05$ ) según la prueba de Tukey.

Tabla 2. Valores comparativos de $\mathrm{CL}_{50}$ con 28 especies acuáticas expuestas al metomilo.

\begin{tabular}{|c|c|c|c|c|}
\hline Grupo & Especie & Duración & $\mathbf{N}^{\circ}$ ensayos & $\mathrm{CL}_{50}$ ug $\mathrm{L}^{-1}$ \\
\hline Pez & Cyprinodon variegatus (Lacépède, 1803 ) & 96 & 2 & 1060 \\
\hline Pez & Ictalurus punctatus (Rafinesque, 1818) & 96 & 9 & 320 \\
\hline Pez & Lepomis macrochirus Rafinesque, 1819 & 96 & 18 & 850 \\
\hline Pez & Micropterus salmoides (Lacépède, 1802) & 96 & 4 & 1005 \\
\hline Pez & Oncorhynchus clarki (Richardson, 1836) & 96 & 2 & 5425 \\
\hline Pez & Oncorhynchus mykiss Walbaum, 1792 & 96 & 19 & 1600 \\
\hline Pez & Pimephales promelas Rafinesque, 1820 & 96 & 10 & 1800 \\
\hline Pez & Salmo salar (Linnaeus 1758) & 96 & 6 & 1200 \\
\hline Pez & Salvelinus fontinalis (Mitchill, 1814) & 96 & 5 & 1500 \\
\hline Pez & $\begin{array}{l}\text { Pseudorasbora parva } \\
\text { (Temminck et Schlegel, 1846) }\end{array}$ & 96 & 1 & 425 \\
\hline Pez & $\begin{array}{l}\text { Corydoras arcuatus } \\
\text { Elwin, } 1939 \text { (Este trabajo, 2008) }\end{array}$ & 48 & 1 & $<2$ \\
\hline Insecta & Chironomus plumosus Linnaeus, 1758 & 96 & 1 & 32 \\
\hline Insecta & Isogenus Newman, $1833 \mathrm{sp.}$ & 96 & 2 & 186 \\
\hline Insecta & Pteronarcella badia (Hagen, 1874). & 96 & 6 & 60 \\
\hline Insecta & Skwala sp. Ricker & 96 & 3 & 29 \\
\hline Crustácea & Daphnia magna (Straus, 1820) & 48 & 8 & 8,8 \\
\hline Crustácea & Acartia tonsa Dana 1849 & 96 & 1 & 410 \\
\hline Crustácea & Cyclops strenuus (Fischer 1851). & 96 & 1 & 190 \\
\hline Crustácea & Eurytemora affinis (Poppe, 1880) & 96 & 1 & 290 \\
\hline Crustácea & $\begin{array}{l}\text { Gammarus italicus } \\
\text { Goedmakers \& Pinkster, } 1977\end{array}$ & 96 & 1 & 47 \\
\hline Crustácea & Gammarus pseudolimnaeus Bousfield, 1958 & 96 & 6 & 920 \\
\hline Crustácea & Gammarus pulex (Linnaeus, 1758) & 96 & 1 & 760 \\
\hline Crustácea & Palaemonetes vulgaris (Say, 1818) & 96 & 1 & 49 \\
\hline Crustácea & Procambarus acutus (Girard 1852) & 96 & 1 & 1000 \\
\hline Mollusca & Bulinus truncatus (Audouin, 1827) & 96 & 1 & 870 \\
\hline Mollusca & Cipangopaludina malleata (Reeve, 1863 ) & 96 & 1 & 25000 \\
\hline Mollusca & Indoplanorbis exustus Deshayes 1834 & 96 & 1 & 6600 \\
\hline Mollusca & Physella acuta (Draparnaud,1805) & 96 & 1 & 18000 \\
\hline Mollusca & Semisulcospira libertina (Gould, 1859) & 96 & 1 & 12000 \\
\hline
\end{tabular}


Tabla 3. Parámetros biométricos y de recuento leucocitario de varias especies de Corydoras.

\begin{tabular}{lcccc}
\hline \multicolumn{1}{c}{ Parámetros } & $\begin{array}{c}\text { Las } 3 \\
\text { especies de } \\
\text { Corydoras }\end{array}$ & C. leucomelas & C. arcuatus & C. leopardos \\
\hline Longitud total (cm) & 4.9 & 4.8 & 6 & 5.1 \\
Longitud estándar (cm) & 3.7 & 3.5 & 4.6 & 4.1 \\
Peso (g) & 2.9 & 2.9 & 4.1 & 3.01 \\
\% Linfocitos (Giemsa) & 64.8 & 63.5 & ND & 69 \\
\% Linfocitos (Wright) & 64.4 & 62.3 & 61 & 76 \\
\% Monocitos (Giemsa) & 18.6 & 17.9 & ND & 22.7 \\
\% Monocitos (Wright) & 18.1 & 18.9 & 11 & 15.8 \\
\% Neutrofilos (Giemsa) & 8.2 & 9.7 & ND & 2.2 \\
\% Neutrofilos (Wright) & 8.1 & 9.2 & 6 & 2.8 \\
\% Eosinófilos (Giemsa) & 4.9 & 5.6 & ND & 3.0 \\
\% Eosinófilos (Wright) & 5.5 & 6.5 & 0 & 1.4 \\
\% Basófilos (Giemsa) & 3.3 & 3.2 & 6 & 3.0 \\
\% Basófilos (Wright) & 3.1 & 2.2 & 22 & 4.0 \\
Macho (Nro.) & 13 & 10 & 1 & 2 \\
Hembra (Nro.) & 7 & 2 & 0 & 5 \\
Indiferenciado (Nro.) & 14 & 14 & 0 & 0 \\
Especimenes (Nro.) & 34 & 26 & 1 & 7 \\
\hline
\end{tabular}

Tabla 4. Comparación entre tres parámetros biométricos, número de neutrófilos y el sexo de Corydoras spp. F = Estadístico de Fisher. $\mathrm{P}=$ Probabilidad.

\begin{tabular}{|c|c|c|c|c|c|}
\hline \multirow[t]{2}{*}{ Parámetros } & \multicolumn{3}{|c|}{ Sexo } & \multirow[t]{2}{*}{$\mathbf{F}$} & \multirow[t]{2}{*}{$\mathbf{P}$} \\
\hline & $\begin{array}{l}\text { Macho } \\
(n=13)\end{array}$ & $\begin{array}{c}\text { Hembras } \\
(\mathbf{n}=7)\end{array}$ & $\begin{array}{c}\text { Indiferenciados } \\
\qquad(\mathrm{n}=14)\end{array}$ & & \\
\hline Longitud total (cm) & $5.15 b$ & $5.50 \mathrm{~b}$ & $4.62^{\mathrm{a}}$ & 8.62 & 0.001 \\
\hline Longitud estándar $(\mathrm{cm})$ & $3.78 \mathrm{ab}$ & $4.22 b$ & $3.46^{\mathrm{a}}$ & 6.63 & 0.004 \\
\hline Peso $(\mathrm{g})$ & $3.35 b$ & $3.56 \mathrm{~b}$ & $2.35^{\mathrm{a}}$ & 8.80 & 0.001 \\
\hline Neutrófilos (Giemsa) (\%) & $14.5 \mathrm{c}$ & $1.80 \mathrm{a}$ & $5 b$ & 6.57 & 0.006 \\
\hline Neutrófilos (Wright) (\%) & $13.5 \mathrm{c}$ & $0.00 \mathrm{a}$ & $6.28 b$ & 4.22 & 0.025 \\
\hline
\end{tabular}

Tabla 5. Comparación del \% leucocitario de Corydoras spp. según técnica de tinción empleada. Solo se incluyeron 24 datos pareados, debido a que en el resto de los recuentos leucocitarios solo se obtuvo una sola lámina de Giemsa o de Wright. $\mathrm{t}=\mathrm{t}$ de Student para datos pareados. P. = Probabilidad.

\begin{tabular}{|c|c|c|c|c|}
\hline $\begin{array}{c}\% \\
\text { Leucocitario } \\
\end{array}$ & $\begin{array}{l}\text { Tinción } \\
\text { Giemsa }\end{array}$ & $\begin{array}{l}\text { Tinción } \\
\text { Wright }\end{array}$ & $t$ & $\mathbf{P}$ \\
\hline Linfocito & 64.2 & 67.3 & 0.89 & 0.37 \\
\hline Monocito & 18.9 & 16.9 & 0.88 & 0.38 \\
\hline Neutrófilo & 8.3 & 7.7 & 0.46 & 0.64 \\
\hline Eosinófilo & 5.0 & 4.4 & 0.37 & 0.70 \\
\hline Basófilo & 3.3 & 2.6 & 0.69 & 0.49 \\
\hline
\end{tabular}

${ }^{1}$ Laboratorio de invertebrados. Facultad de Ciencias Biológicas. Universidad Ricardo Palma. joseiannacone@yahoo.es

${ }^{2}$ Laboratorio de Ecofisiología Animal. Facultad de Ciencias Naturales y Matemáticas. Universidad Nacional Federico Villarreal. lalvarino@sedapal.com.pe 